\title{
MS012.P06
}

\section{True molecular structure of ixazomib citrate}

Eliska Skorepova ${ }^{1}$, Igor Cerna $^{2}$, Ruzena Vlasakova ${ }^{2}$, Vit Zvonicek $^{1}$

${ }^{1}$ University Of Chemistry And Technology Prague, Prague 6, Czech Republic, ${ }^{2}$ Zentiva k.s., Prague, Czech Republic E-mail: eliska.skorepova@vscht.cz

Ixazomib citrate is a very recently approved anti-cancer drug. In all currently available literature, the molecule is characterized as containing a borate cycle formed when ixazomib is reacted with citric acid to form a stabilized ixazomib citrate that can be administered orally. However, in this work, we will show that none of the up-to-date presented structural formulas of ixazomib citrate are fully accurate. Through the combination of single-crystal and powder X-ray diffraction, solid-state NMR and DFT quantum mechanical calculations we show that, in addition to the citrate ring, another 5-membered ring is formed. These two rings are connected by the boron atom, making this compound a spirocyclic borate.

We would like to acknowledge support from the Grant Agency of the Czech Republic (project no. 17-23196S).

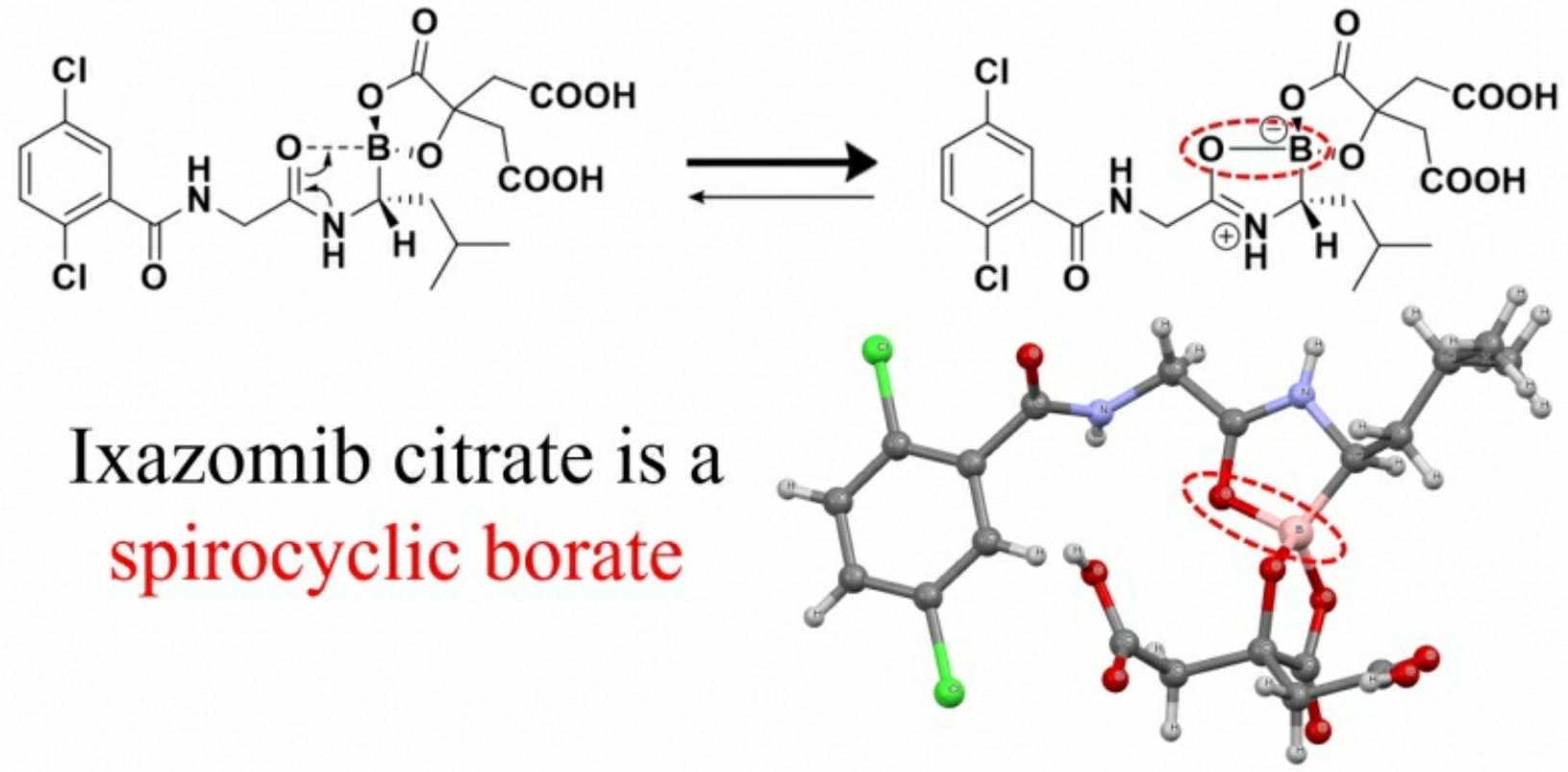

Keywords: molecular structure, pharmaceutical, 